\title{
BMJ Open Interventions promoting physical activity among adults and children in the six Gulf Cooperation Council countries: protocol for a systematic review
}

\author{
Fiona Pearson (D) , ${ }^{1}$ Peijue Huangfu, ${ }^{2}$ Farah M Abu-Hijleh, ${ }^{3}$ Susanne F Awad, ${ }^{4}$ \\ Laith J Abu-Raddad (D) ,4,5 Julia A Critchley ${ }^{2}$
}

To cite: Pearson F, Huangfu $P$, Abu-Hijleh FM, et al. Interventions promoting physical activity among adults and children in the six Gulf Cooperation Council countries: protocol for a systematic review. BMJ Open 2020;10:e037122. doi:10.1136/ bmjopen-2020-037122

- Prepublication history and additional material for this paper are available online. To view these files, please visit the journal online (http://dx.doi. org/10.1136/bmjopen-2020037122).

Received 20 January 2020 Revised 22 May 2020 Accepted 03 July 2020

Check for updates

(C) Author(s) (or their employer(s)) 2020. Re-use permitted under CC BY-NC. No commercial re-use. See rights and permissions. Published by BMJ.

For numbered affiliations see end of article.

Correspondence to

Dr Fiona Pearson;

fiona.pearson2@ncl.ac.uk

\section{ABSTRACT}

Introduction Prevalence of overweight, obesity and diabetes are high and rising across the Gulf Cooperation Council (GCC) countries (Oman, Bahrain, Kuwait, Qatar, Saudi Arabia and the United Arab Emirates). In parallel, physical activity (PA) levels are low relative to international standards. PA aids weight control and reduces risk of non-communicable diseases including diabetes and cardiovascular disease. It is likely interventions developed elsewhere will not translate to GCC countries due to unique environmental, social and cultural factors. This protocol is for a systematic review assessing the efficacy of interventions promoting PA within GCC countries among generally healthy adults and children. The primary outcome of interest is change in objectively measured or self-reported PA levels, the secondary outcomes of interest are changes in anthropometry or chronic disease risk factors (eg, blood pressure). Interventions will be compared with no intervention or those of differing PA intensity or duration. The relationships between PA change and the following will be assessed: intervention intensity or duration, season in which intervention occurs, sex, age, nationality and sustainability over time.

Methods and analysis A systematic search strategy will identify indexed publications on the efficacy of interventions promoting PA. Randomised controlled trials and quasi-experimental studies recruiting predominantly healthy children and adults will be included. Studies of exercise rehabilitation will be excluded. Medline, Embase, Cinahl, Cochrane Library, SportDiscus, Web of Science, Index Medicus for the Eastern Mediterranean Region and Qscience will be searched. Clinical trial registries including the International Clinical Trials Registry Platform, the Iranian Registry of Clinical Trials and ClinicalTrials. gov will be searched for ongoing and unpublished studies. Searches will be ran from database inception until 1 May 2020 and be supplemented by checking references of key articles. Two reviewers will independently screen identified citations then full texts using prespecified inclusion and exclusion criteria. Piloted data extraction forms will be used in duplicate. Inconsistencies in screening or data extraction will be resolved by a third investigator or study author contact. Risk of bias will be independently assessed
Strengths and limitations of this study

- The methodology of this systematic review follows a standard approach and is being made transparent apriori with registration on PROSPERO and publication within BMJ Open.

- Risk of bias is being assessed for each study using a validated tool appropriate for each study's design. As well as this, the Grading of Recommendations, Assessment, Development and Evaluations approach is being used to assess the certainty of the evidence contributing to each outcome being reported.

- Appropriate analysis plans have been specified based on the evidence identified. For example, metaanalysis will only be completed contingent on the level of clinical diversity and risk of bias identified.

- The search strategy for grey literature in this review is limited; however, regional databases and relevant clinical trial registries have been included.

- Given the aim of the review, geographical setting has been made an inclusion and exclusion criteria. Although this decision was made to align with the review's primary aim, it means there will be no ability to compare intervention efficacy across more heterogeneous geographical settings.

by two reviewers using validated tools. A narrative summary of findings will be produced supplemented with meta-analyses and exploration of heterogeneity as appropriate.

Ethics and Dissemination The review aims to strengthen the findings of the primary studies it incorporates and explore the impact of setting. It will synthesise existing published aggregate patient data. If publications or data with ethical concerns are identified, they will be excluded from the review. Results of the systematic review will be published in full and authors will engage directly with research audiences and key stakeholders to share findings.

PROSPERO registration number 131817. 


\section{BACKGROUND}

The prevalence of overweight and obesity among adults residing in the six Gulf Cooperation Council (GCG) countries (Oman, Bahrain, Kuwait, Qatar, Saudi Arabia and the United Arab Emirates) is very high ranging from $69 \%$ to $86 \% .^{1-3}$ In addition, obesity and alarmingly type 2 diabetes mellitus (T2DM) are becoming increasingly common in the child and adolescent population (<18 years old). ${ }^{45}$ Overweight and obesity are chronic health statuses associated with high morbidity and mortality rates. In particular, increased body mass index (BMI) is a major risk factor for cardiovascular disease, diabetes mellitus, musculoskeletal disorders and cancer. ${ }^{67} \mathrm{BMI}$ is largely influenced by energy intake and calorie control, but increasing physical activity can contribute to increased weight loss and weight maintenance. ${ }^{8}$ Evidence indicates that physical activity (PA) can improve cardiovascular fitness and insulin sensitivity, reduce incidence of T2DM, improve glycaemic control for people living with T2DM, lower blood pressure, lower risk of depression and anxiety, ${ }^{9}$ and reduce risks of certain cancers. ${ }^{10} \mathrm{PA}$ can also improve cognitive function and reduce risks of dementia. ${ }^{10}$

PA is defined as any bodily movement produced by skeletal muscles that requires energy expenditure. ${ }^{1011}$ PA includes different forms of exercise (activities that are planned and deliberately performed to improve physical fitness) ${ }^{12}$ as well as many activities that are completed as part of routine daily life such as active transportation, household chores and other recreational activities. PA is often described using 'FITT' principles (Frequency, Intensity, Time and Type) with most intervention studies aiming to increase at least moderate or vigorous activities (activities expending at least three times more energy than at rest). ${ }^{10}$ Current WHO recommendations for PA levels among adults are to reach a target level of $150 \mathrm{~min}$ or more of moderate PA per week, or 75 min or more of vigorous activities (or a combination of the two $)^{13}$ and 1 hour a day for children (aged 5-17). ${ }^{12}$ However, there is evidence that any increase in PA, even below these levels, improves cardiovascular health and reduces diabetes risk. ${ }^{12} 14$

There appears to be a lack of objectively measured data on PA in the GCC countries ${ }^{15}$ and only limited data on self-reported PA levels. ${ }^{12}$ However, data from the WHO Global Observatory suggest that the Eastern Mediterranean region has the highest regional prevalence of physical inactivity among both adults $(31 \%)$ and adolescents $(87 \%) .{ }^{10} \mathrm{PA}$ levels are thought to be even lower across the GCC due to a range of interrelated region specific factors including: climate, lack of facilities and infrastructure to promote PA (eg, pavements and walking routes), high levels of traffic, lack of parental support for children to be physically active and, for women, cultural expectations surrounding PA behaviour. ${ }^{1617}$ A recent review from the Arab region (which includes the GCC countries) found that over $40 \%$ of adults were physically inactive in most countries, and activity levels among women were generally lower than men. ${ }^{17}$ Activity levels in children were also lower than the global average. ${ }^{17}$ Given both the low current levels and regional specific factors, it is thought that interventions promoting PA developed and evaluated for populations elsewhere (most often Northern Europe or North America but even in neighbouring countries) may not be appropriate to implement in the Gulf region without adjusting for contextual factors and examining regional evidence in practice.

A previous narrative review published in 2016 identified six studies describing PA interventions targeted at adults; however, it did not use a very comprehensive systematic search strategy, or state clear inclusion and exclusion criteria. ${ }^{15}$ Most of the six studies identified were not randomised controlled trials (RCTs) and had substantial limitations including small sample sizes and a lack of long-term follow-up. ${ }^{15}$ The review identified that greater priority should be given to increasing intervention research to guide national policy and programmes on chronic disease prevention including through use of PA. At the time of the 2016 review, no studies of physical activity promotion were identified among children, but some school-based studies are now available. ${ }^{18} 19$ We will stratify results by age group (adults or children<16) to reflect this important updated information. This planned systematic review will include a more comprehensive search likely to identify studies previously uncaptured within the narrative review, as well as more recently published studies ${ }^{18} 19$ leading to further insights being gained from appraising the expanding evidence base.

This protocol is for a systematic review of the efficacy of interventions that aim to promote any form of physical activity undertaken within the six GCC countries among healthy free-living adults and children. Both postintervention and pre-intervention control groups will be included. The review will assess the efficacy of any intervention compared with minimal (eg, brief one-off advice) or no intervention at: increasing PA levels from baseline, improving cardiovascular and/or metabolic fitness or improving anthropometric markers (such as BMI). If sufficient numbers of studies are identified, secondary aims will be to assess whether longer or more intensive interventions (more contacts or sessions per week) result in further increases in PA. If later follow-up data are available, we will assess whether intervention benefits are maintained beyond the end of the intervention period. If feasible, we will also perform subgroup analyses to assess differences in PA outcomes between men and women. We will perform sensitivity analyses to explore which types of intervention appear to be most effective among children (<16 years) and adults separately, and also among Gulf state nationals as well as migrant populations separately. If sufficient studies are identified, we will also explore the effect of seasonality (spring, autumn, summer or winter) on intervention efficacy. 
METHODS AND ANALYSIS

\section{Inclusion criteria}

We will include RCTs and any observational studies that are quasi-experimental in design. This will include any observational studies with a comparator group and also difference-in-difference (pre-post-intervention) studies, interrupted time series studies and propensity score matching studies. We will include studies published in English and in Arabic, and do not anticipate identifying studies published in any other language.

\section{Participants}

Children and adults irrespective of nationality or non-communicable diseases (NCD) risk factor status, including migrant populations living in the six GCC countries. Any trials specifically targeted at patients requiring physical exercise for rehabilitation (eg, after a stroke, myocardial infarction, joint replacement surgery) or pulmonary rehabilitation (eg, patients with Chronic Obstructive Pulmonary Disease) will not be included as exercise capacity would likely be substantially different in these groups and distinct supervised approaches may therefore be required.

\section{Intervention}

Any form of PA intervention, whether supervised or unsupervised, offered on a repetitive basis over any predefined period of time, either in isolation or in combination with other intervention components as part of broader lifestyle interventions. Types of physical activity programme will not be prespecified but might include: web-based or mobile phone-based prompts and reminders, groupbased exercise sessions, walking programmes taking place in the community or in workplaces and schools, motivational messaging and counselling. Studies evaluating only brief advice (ie, one-off advice from healthcare practitioners or others) to increase PA will be excluded, except when this forms part of the 'comparator' group.

\section{Comparison group}

The PA intervention will be compared with no intervention, to a control offering brief one-off advice to increase $\mathrm{PA}$, to a control containing no PA components, or to a control offering PA at different levels of exertion, intensity or duration. Pretest and post-test groups will also be included.

\section{Outcome}

The primary outcome of interest is a change in level of PA, measured by self-reporting (through validated questionnaires such as the International Physical Activity Questionnaire) $)^{20} 21$ or through more objective methods such as accelerometers, heart rate monitors or step counters. Objective methods of measurement are preferred as there is some evidence that self-reported measurements can significantly overestimate PA levels compared with more objective assessments such as by accelerometers, ${ }^{22}$ though this has not been a consistent finding. ${ }^{23}{ }^{24}$ Quantitative data are likely to be available as mean changes in
PA levels (self-reported or objective) or potentially the proportion of the intervention and control arms meeting a fixed level of PA (such as 150 min of moderate activity or more per week), or participating in group-based sessions or specific activities.

Secondary outcomes of interest are weight change at any time point, other indicators of body mass and anthropometry (eg, BMI, waist circumference, waist-tohip ratio), and assessment of other cardiovascular and metabolic risk markers (eg, blood pressure, blood lipids). Other process outcomes such as dropping out from the programme, and adherence to group exercise sessions will be reported where available.

\section{Setting}

'Healthy' non-institutionalised adults and children living in the community in the six GCC countries. Interventions can take place in any setting whether it is home based, centre based, community based, worksite or school based.

\section{Search methods}

A systematic search strategy will be used to identify a broad range of published PA intervention studies among adults and children. Searches will be conducted from database inception to May 2020 in Medline, Embase, Cinahl, Cochrane Library, SportDiscus and Web of Science using the proposed search terms set out in online supplementary appendix 1 translated for each database. We will also search key regional databases and journals including Index Medicus for the Eastern Mediterranean Region (IMEMR), Qscience (a database in Qatar) and Saudi Medical Journal. We will search trial registries for information on any unpublished but potentially relevant trials including the International Clinical Trials Registry Platform, the Iranian Registry of Clinical Trials and ClinicalTrials.gov. Database searches will be supplemented by reference and citation checking of key articles, and contact with experts.

\section{Data management}

All data will be stored centrally and securely using institutional servers. The review process will be documented and an annotated Preferred Reporting Items for Systematic Reviews and Meta-Analyses flow diagram will be produced depicting the number of records initially identified through the different database searches and other forms of searching, the number of records included and excluded at each stage of the process and the reasons for any exclusions.

Initial citation sifting will be completed independently by two investigators in EndNote V.X7 using outlined inclusion and exclusion criteria. A $10 \%$ pilot of sifting will be carried out to assess whether any amendments are required to inclusion/exclusion criteria.

As needed, publications will be retrieved in full and a prespecified, tested Excel form will be used to assess inclusion or exclusion of full text publications. Where reviewers disagree on inclusion or exclusion, a third 
independent reviewer will be consulted or disagreement will be resolved by discussion.

\section{Data extraction}

A prespecified, tested Excel form will be used for data extraction including data on quality and risk of bias. Piloting of this data extraction tool will occur in a subsample of included studies before the full extraction is carried out. Data on the following will be extracted independently by two investigators: publication characteristics, trial characteristics (design, duration, randomisation, allocation concealment, blinding), intervention (exercise type/frequency/duration/intensity, comparison, method of delivery, season of delivery), participants (gender, age, nationality, definition of overweight or obesity, baseline characteristics, assessment of compliance or relapse, withdrawals or losses to follow-up), subgroups, outcome/ results (activity level, marker of weight loss), other events captured (eg, injuries) and length of follow-up.

Data extraction will be completed independently by two investigators and any discrepancies in extraction will be resolved through consultation of a third independent reviewer. If at any point in the review process, data are missing from a primary publication or are unclear, study authors will be contacted for clarification.

\section{Assessment of methodological quality}

To give a study-centric risk of bias summary, the following validated tools will be used: for observational studies, the Newcastle Ottawa Score ${ }^{25}$ or ROBINS- ${ }^{26}$; and for RCTs, Cochrane Risk of Bias 2. ${ }^{27}$ To highlight the certainty of evidence contributing to each outcome Grading of Recommendations, Assessment, Development and Evaluations (GRADE), a validated approach, will be used. ${ }^{13} 28$ GRADE incorporates its own outcome-centric risk of bias assessment. Risk of bias assessment and GRADE will also be conducted by two independent reviewers, with any disagreements resolved through a third party.

\section{Strategy for data synthesis}

The characteristics of each study and intervention will be collated in a 'characteristics of included studies' table. If statistical synthesis of results is not appropriate, a structured report of the effects will be given. The difference in self-reported physical activity, or objectively measured physical activity (eg, step counts), or uptake of groupbased sessions, or participation in specific activities will be compared between intervention or comparator groups. If statistical pooling is feasible, fixed or random effects Mantel-Haenszel models will be produced dependent on the heterogeneity present between studies. Heterogeneity will be assessed using the $\chi^{2}$ test, the $\mathrm{I}^{2}$ statistic and the prediction interval. Given the broad clinical and methodological inclusion criteria, it is likely that a random effects model will be used. Meta-analyses will be completed using STATA V.15 with $\log$ files of all sessions stored. If plausible (ie, if there are sufficient studies overall in each subcategory) meta-analyses and subgroup analyses will be completed by intervention type, age (adults or children), sex, nationality, initial weight, comorbidities and levels of adherence.

Using subgroup analysis or meta-regression as appropriate, potential causes of heterogeneity will be explored including: the site of intervention (eg, schools, workplaces, clinic or population based), multicomponent interventions (eg, dietary advice, smoking cessation, or focussing exclusively on physical activity), mode of delivery (eg, online, face to face), intensity and duration of the intervention (such as number of contacts with advisors or number of exercise sessions offered) and attrition bias (losses to follow-up in each intervention arm $<20 \%$, intention-to-treat analyses performed).

If sufficient numbers of studies are identified, sensitivity analyses will be completed to explore whether study design, study size, study quality, study duration, study setting, intervention type, or duration and intensity of exercise impact pooled intervention effect estimates. Participant characteristics (eg, presence of comorbidities, average baseline weight, age group (children or adults), biological sex) intervention features, season of intervention and different modes of delivery of the intervention will also be noted and considered in sensitivity analyses.

Publication bias will be assessed using funnel plots if more than 10 studies are being included within a metaanalysis; Egger's test for bias will also be performed.

\section{Patient and public involvement statement}

This protocol was designed without patient involvement. Patients were not invited to comment on the study design and were not consulted to develop patient relevant outcomes or interpret the results. Patients were not invited to contribute to the writing or editing of this document for readability or accuracy.

\section{ETHICS AND DISSEMINATION}

Given the nature of the systematic review process, ethical and safety considerations are minimal. No concerns are held relating to the reuse of patient data for new research or the need to seek further informed consent. The review will collate, synthesise and present previously published aggregate patient data. No concerns are held relating to patient confidentiality. There are no plans for data deposition or curation. If publications or data with ethical concerns are identified by the review, although unlikely, they will be excluded. Any amendments made to the protocol subsequent to its publication will initially be highlighted on PROSPERO and indicated with a date. They will then be provided as a dated addendum to this manuscript. Findings will be published in a relevant academic journal and authors will engage with researchers through academic fora and key stakeholders.

\section{Author affiliations}

${ }^{1}$ Evidence Synthesis Group, Population Health Research Institute, Newcastle University, Newcastle Upon Tyne, UK

${ }^{2}$ Population Health Research Institute, St George's University of London, London, UK 
${ }^{3}$ Department of Public Health, College of Health Sciences, Academic Quality Affairs Office, QU Health, Qatar University, Doha, Qatar

${ }^{4}$ Infectious Disease Epidemiology Group, Weill Cornell Medical College, Qatar, Cornell University, Qatar Foundation - Education City, Doha, Qatar ${ }^{5}$ Department of Healthcare Policy and Research, Weill Cornell Medicine, Cornell University, New York, New York, USA

Contributors JAC and FP conceptualised the study. FP and JAC designed the study protocol and drafted the manuscript. All authors (FP, JAC, PH, FMA-H, SFA and LJA-R) were involved in critically revising both the protocol for this systematic review and the drafted manuscript.

Funding This publication was made possible by NPRP grant 10-1208-160017 from the Qatar National Research Fund (a member of Qatar Foundation). The findings achieved herein are solely the responsibility of the authors.

Disclaimer Neither funder, sponsor nor the author's institutions played a role in developing the protocol. Details herein are solely the responsibility of the authors.

Competing interests None declared.

Patient and public involvement Patients and/or the public were not involved in the design, or conduct, or reporting, or dissemination plans of this research.

Patient consent for publication Not required.

Provenance and peer review Not commissioned; externally peer reviewed.

Open access This is an open access article distributed in accordance with the Creative Commons Attribution Non Commercial (CC BY-NC 4.0) license, which permits others to distribute, remix, adapt, build upon this work non-commercially, and license their derivative works on different terms, provided the original work is properly cited, appropriate credit is given, any changes made indicated, and the use is non-commercial. See: http://creativecommons.org/licenses/by-nc/4.0/.

\section{ORCID iDs}

Fiona Pearson http://orcid.org/0000-0003-1626-0862

Laith J Abu-Raddad http://orcid.org/0000-0003-0790-0506

\section{REFERENCES}

1 Awad SF, O'Flaherty M, Critchley J, et al. Forecasting the burden of type 2 diabetes mellitus in Qatar to 2050: a novel modeling approach. Diabetes Res Clin Pract 2018;137:100-8.

2 Al-Quwaidhi AJ, Pearce MS, Critchley JA, et al. Trends and future projections of the prevalence of adult obesity in Saudi Arabia, 19922022. East Mediterr Health J 2014;20:589-95.

3 Kilpi F, Webber L, Musaigner A, et al. Alarming predictions for obesity and non-communicable diseases in the middle East. Public Health Nutr 2014;17:1078-86.

$4 \mathrm{Al} \mathrm{Hammadi} \mathrm{H,} \mathrm{Reilly} \mathrm{J.} \mathrm{Prevalence} \mathrm{of} \mathrm{obesity} \mathrm{among} \mathrm{school-age}$ children and adolescents in the Gulf cooperation Council (GCC) states: a systematic review. BMC Obes 2019;6:3.

5 Nahhas MA, Asamoah F, Mullen S, et al. Epidemiology of overweight and obesity in early childhood in the Gulf cooperation Council countries: a systematic review and meta-analysis protocol. BMJ Open 2018;8:e019363.

6 Guh DP, Zhang W, Bansback N, et al. The incidence of comorbidities related to obesity and overweight: a systematic review and meta-analysis. BMC Public Health 2009;9:88.
7 Pi-Sunyer $X$. The medical risks of obesity. Postgrad Med 2009;121:21-33.

8 Cox CE. Role of physical activity for weight loss and weight maintenance. Diabetes Spectr 2017;30:157-60.

9 Warburton DER, Nicol CW, Bredin SSD. Health benefits of physical activity: the evidence. CMAJ 2006;174:801-9.

10 Rhodes RE, Janssen I, Bredin SSD, et al. Physical activity: health impact, prevalence, correlates and interventions. Psychol Health 2017:32:942-75.

11 World Health Organization. Global strategy on diet, physical activity and health. Available: https://www.who.int/dietphysicalactivity/pa/ en/: Switzerland [Accessed 6 Mar 2020].

12 World Health Organisation. Global recommendations on physical acitivty for health. Switzerland, 2010.

13 Piercy KL, Troiano RP, Ballard RM, et al. The physical activity guidelines for Americans. JAMA 2018;320:2020-8.

14 Warburton DER, Bredin SSD. Health benefits of physical activity: a systematic review of current systematic reviews. Curr Opin Cardiol 2017;32:541-56.

15 Mabry R, Koohsari MJ, Bull F, et al. A systematic review of physical activity and sedentary behaviour research in the oilproducing countries of the Arabian Peninsula. BMC Public Health 2016;16:1003.

16 Althoff T, Sosič R, Hicks JL, et al. Large-Scale physical activity data reveal worldwide activity inequality. Nature 2017;547:336-9.

17 Sharara E, Akik C, Ghattas H, et al. Physical inactivity, gender and culture in Arab countries: a systematic assessment of the literature. BMC Public Health 2018;18:639-39.

18 Choudhury S, Omar O, Arora T, et al. Qatar obesity reduction study (QORS): report on a pilot school-based nutrition education campaign in Qatar. J Child Obes 2018;S2.

19 Allafi AR. Effects of rewards and pedometer-feedback on children's physical activity: a school-based intervention study. Progr Nutr 2019;22.

20 Lee PH, Macfarlane DJ, Lam TH, et al. Validity of the International physical activity questionnaire short form (IPAQ-SF): a systematic review. Int J Behav Nutr Phys Act 2011;8:115.

21 Fogelholm M, Malmberg J, Suni J, et al. International physical activity questionnaire: validity against fitness. Med Sci Sports Exerc 2006;38:753-60.

22 Joint Health Surveys Unit. Health survey for England 2008: physical activity and fitness. The Information Centre: Leeds, 2010.

23 Prince SA, Adamo KB, Hamel ME, et al. A comparison of direct versus self-report measures for assessing physical activity in adults: a systematic review. Int J Behav Nutr Phys Act 2008;5:56.

24 Sabia S, van Hees VT, Shipley MJ, et al. Association between questionnaire- and accelerometer-assessed physical activity: the role of sociodemographic factors. Am J Epidemiol 2014; 179:781-90.

25 Wells GA, Shea B, O'Connell D, et al. The Newcastle-Ottawa scale (NOS) for assessing the quality of nonrandomised studies in metaanalyses, 2019. Available: http://www.ohri.ca/programs/clinical_ epidemiology/oxford.asp

26 Sterne JA, Hernán MA, Reeves BC, et al. ROBINS-I: a tool for assessing risk of bias in non-randomised studies of interventions. BMJ 2016;355:i4919.

27 Sterne JAC, Savović J, Page MJ, et al. Rob 2: a revised tool for assessing risk of bias in randomised trials. BMJ 2019;366:14898.

28 Brożek JL, Akl EA, Alonso-Coello P, et al. Grading quality of evidence and strength of recommendations in clinical practice guidelines. Allergy 2009;64:669-77. 